Article

\title{
Reducing the Risks during the Purchase of Five-Axis CNC Machining Centers Using AHP Method and Fuzzy Systems
}

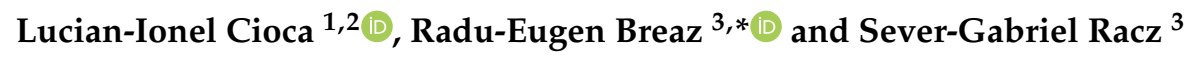 \\ 1 Department of Engineering and Management, Engineering Faculty, Lucian Blaga University of Sibiu, \\ Victoriei 10, 550024 Sibiu, Romania; lucian.cioca@ulbsibiu.ro \\ 2 Academy of Romanian Scientists, 010071 Bucharest, Romania \\ 3 Department of Industrial Machines and Equipment, Engineering Faculty, Lucian Blaga University of Sibiu, \\ Victoriei 10, 550024 Sibiu, Romania; gabriel.racz@ulbsibiu.ro \\ * Correspondence: radu.breaz@ulbsibiu.ro; Tel.: +40-745-374-776
}

Received: 4 December 2018; Accepted: 26 December 2018; Published: 9 January 2019

check for updates

\begin{abstract}
Nowadays, companies are in the process of renewing their manufacturing lines by equipping them with modern five-axis CNC (computer numerical control) machining centers. The decision to select between different five-axis CNC machining centers, with similar technological capabilities is a difficult process, so the main goal of this work was to develop a method for assisting it. The proposed approach relies on seven technical criteria, four quantitative ones (traverse speed, thrust, spindle power, and spindle speed) which can be expressed by crisp numerical values, while the other three (flexibility, operation easiness, and setup time) are qualitative ones. The analytic hierarchy process (AHP) was used for ordering four variants of five-axis CNC milling machining centers. The qualitative criteria were processed using fuzzy systems to be expressed by crisp numerical values, suitable for AHP. Finally, the four variants of five-axis CNC milling machining centers were hierarchized and the best one was chosen. A sensitivity analysis was also unfolded to certify the robustness of the AHP.
\end{abstract}

Keywords: AHP; five-axis CNC milling machining centers; equipment selection; fuzzy systems

\section{Introduction}

Proper equipment selection highly influences the production costs and the products quality, which are paramount for customer satisfaction. One of the most important technological equipment is the machine-tool, which represents the backbone of manufacturing industry. Selecting between different alternatives of machine-tools is a very complex process, a multiple criteria decision making one [1], considering the large number of options, with quite similar performances and prices. The prices of these systems differ less and less from one producer to another, consequently price has become a selection criterion which has reduced utility.

Improperly selected machine-tools can affect the manufacturing system performance, by introducing a series of risks related to costs, production quality, productivity [2], market share and environmental aspects [3].

The process of selecting the proper machine-tool is in most of the cases subject of vague information, which has to be processed in order to extract crisp values from it. Thus, fuzzy logic techniques are widely used for this purpose, given their ability to deal extracting significant information from vague data. In [4], a fuzzy technique based upon information axiom was used for selecting between alternative solutions for punching machines (machine-tools for processing sheet metal parts), while in [5] fuzzy techniques were used for material handling equipment selection. A comparison 
between flexible manufacturing systems using fuzzy techniques and axiomatic design was presented in [6].

Analytical hierarchical process (AHP) developed by Saaty $[7,8]$ is a method used for decision making, based upon pairwise comparisons. It allows the use of both measurable inputs and subjective ones, based upon preferences according to human judgement. Fuzzy approach and TOPSIS (technique for order of preference by similarity to ideal solution) were also extensively used for solving decision making problems. The work presented in [9] introduced a novel multi-attribute decision making (MADM) method under interval-valued intuitionistic fuzzy (IVIF) set environment by integrating a (TOPSIS) method. An extended technique for order preference by similarity to ideal solution method under linguistic interval-valued intuitionistic fuzzy set (LIVIFS) has been introduced in [10] and validated by means of a numerical example. An extension of TOPSIS method, based upon set pair analysis, for decision-making application was presented in [11]. An extension of TOPSIS method is developed by combining the proposed connection number for IVIFSs was introduced in [12]. An example has presented to demonstrate the proposed method and to compare it with other existing measures. A Pythagorean fuzzy technique for TOPSIS method by taking the preferences of the experts in the form of interval-valued intuitionistic Pythagorean fuzzy decision matrices has been introduced in [13]. Another application of TOPSIS under cubic intuitionistic fuzzy (CIF) environment for multi-criteria decision making was presented in [14].

AHP was one the most used method for machine-tools selection presented in the literature. In the work presented in [15], which used an AHP approach, four criteria (productivity, flexibility, safety and environment, and adaptability) were used for comparison, together with a series of sub-criteria. Here, some sub-criteria had taken into consideration for comparison the existence of the computer numerical control (CNC) at the machine-tool level. It is noticeable that in 2007, the literature still considers machine-tools without $\mathrm{CNC}$ as an option to be considered. The other sub-criteria are strictly related with technical features of machine-tools and not influenced if the machines-are equipped or not with CNC. The AHP approach is validated by a series of analyses, the sensitivity analysis being one of them. Other approaches using AHP method for equipment selection were presented in [16] (for material handling equipment selection) and [17] (for assembly lines equipment selection). The AHP method was also used for assessing the sustainability of the use phase of different alternatives of machine-tools [18]. Economical (productivity, cost), technical (precision, flexibility), and environmental (power/energy consumption, emission control) criteria were considered to select the best machine-tool system. No reference was made with regards of the influence of CNC equipment upon the selected criteria. Another use of AHP method in the field of industrial equipment was presented in [19], were an approach for selecting between safety devices was introduced.

In order to increase the efficiency of AHP, some hybrid techniques were developed. In [20] fuzzy logic was used in conjunction with AHP for machine-tools selection. Fuzzy matrices were built for the pairwise comparisons. The criteria used for comparison were divided into main attributes (productivity, flexibility, space, adaptability, precision, reliability, safety and environment, and maintenance and service) and attributes (most of them being characteristic features of the machine tools, such as spindle speed, cutting feed, power, number of tools, rotary table). The CNC was considered only by an attribute (CNC type). However, the approach was declared by the authors to be aiming to conventional machine-tools selection, instead of CNC ones.

A combined approach for machine-tools selection, based upon AHP and fuzzy techniques was presented in [21]. Six selection attributes of the analyzed machine-tools alternatives were considered: flexibility, operation easiness, reliability, quality, implementation easiness, and maintainability. A software tool was also developed under MATLAB to support and implement the proposed method.

A hybrid approach, using AHP and preference ranking organization method for enrichment evaluations (PROMETHEE) was proposed in [22]. The considered selection criteria used in this approach were: price, weight, power, spindle (speed), diameter, and stroke. AHP was used to assign 
weights to each criterion, while PROMETHEE was used to determine priorities for the alternative equipment. The approach makes no reference to the CNC character of the analyzed machine-tools.

Another combined approach, based upon fuzzy TOPSIS and fuzzy AHP was introduced in [23] for vertical machining centers (complex machine-tools with numerical control). The selection attributes taken into consideration by this approach were: cost, operative flexibility, installation easiness, maintainability and serviceability, productivity, compatibility, safety and user friendliness. The method was considered by the author effective, especially when conflicting selection attributes are considered.

A TOPSIS based method for machine-tools selection was presented in [24]. In the approach the authors compared the use of fuzzy numbers instead of crisp values and highlighted the advantages of the first ones. However, it was also pointed some situations (low level of fuzziness) were the use crisp numbers will be adequate. Seven criteria of comparison were taken into consideration: table area, spindle speed, power, tool number, tool changing time, maximum tool diameter, and positioning accuracy.

A method of selecting between machining centers based upon a combined fuzzy AHP and TOPSIS multi-criteria approaches was presented in [25]. Among the selection criteria the following were considered: table size, spindle speed, power, number of tools, tool change time, and tool diameter. The combined approach is considered by the authors as a useful tool for improving the selection process.

Fuzzy analytical network process (fuzzy ANP), seen as a generalization of AHP using fuzzy techniques [26], was used in the work presented in [27]. Increased customer satisfaction and increased profitability were considered as determinants for the ANP based network and, as attributes enabling them were used technical characteristics of the machine-tools such as spindle speed, main power, cutting feed, traverse speed, and many others. The CNC system was also considered by its capability (not clearly defined), its reliability and its DNC (direct numerical control—ability to communicate with other computers or $\mathrm{CNC}^{\prime} \mathrm{s}$ ) integration. The proposed method was declared by the authors to be effective, yet cumbersome, in implementation compared to AHP. However, considering the risks (cost, reduced productivity, losing market share) associated to wrong decision related to selecting the proper technological equipment, it was considered that the proposed method should be implemented by the companies

In [28], three methods of equipment selection are comparatively presented and applied: digraph and matrix, AHP, and ANP. The considered selection criteria were: price, weight, power, spindle speed, diameter (of the tool), and stroke. Finally, a milling machine is selected form the proposed alternative and digraph a matrix method is declared to be somehow superior to the other two methods, mostly on terms of simplicity of implementation.

A modified TOPSIS and alpha-cut fuzzy ANP method for machine tool selection was proposed in [29]. The approach is one the first encountered in the literature which took into consideration some aspects related with the computer numerical control $(\mathrm{CNC})$ of the modern machine-tools. Among evaluation attributes, one related to $\mathrm{CNC}$ was considered-CNC type-but it was unclear how was it quantized. The other attributes were close to the ones considered in other approaches: spindle speed, power, cutting feed, traverse speed, number of tools, rotary table, machine dimensions, repeatability, thermal deformation, and many others.

The interaction between selection attributes was taken into consideration in the approach presented in [30]. The proposed method was based upon a hybrid fuzzy ANP and COPRAS-G (COmplex Proportional ASsessment of alternatives with Grey relations). Twelve attributes (among them productivity, flexibility, precision, reliability, and costs), each one with several sub-attributes were considered, similar as in [29]. Again, the CNC character was considered by a single sub-attribute (CNC type). Weight were introduced for each attribute, to express the interactions between them. The method was considered by the authors as promising.

There are also reported in the literature approaches relying on experimental test for selecting the proper machine-tool. The work presented in [31] introduced a method for selecting between high 
speed machine tools based upon performance tests which had targeted the accuracy of the machined parts and other machining process parameters.

Energy consumption optimization is also one of the methods used for machine-tools selection. In [32], a mathematical model relying on this approach was developed. All phases of a machining process (cutting, idle, and setup phases) were considered for the analysis.

Industrial robots are also important technological equipment and method for selecting between alternative robotic structures were also developed [33-36]. An integrated approach, based upon fuzzy Delphi method (FDM), fuzzy AHP (FAHP), fuzzy modified TOPSIS or fuzzy VIKOR, and Brown-Gibson model is presented in [36].

\section{Basic Concepts and Objectives}

CNC (computer numerical control) equipment adds many capabilities to metal cutting machine-tools. By combining the CNC equipment with the mechanical structure of a machine-tools (frame, machine slides, transmission systems, actuation systems, main spindle, tools and tool-changing systems) the accuracy, productivity and flexibility of the machine increase dramatically. Moreover, the machining process became entirely automatic and it is controlled by a computer program (machining code) which runs on the CNC equipment. The combination between the machine-tool and the CNC equipment is called CNC machine-tool, or CNC machining centers, Figure 1.

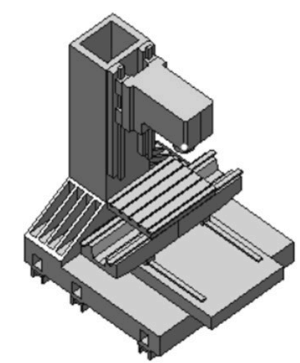

Machine-tool
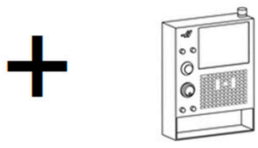

CNC equipment

Figure 1. CNC machine-tool as combination between machine-tool structure and CNC equipment.

CNC machine-tools are usually built in a modular way. The mechanical structure is assembled 'in-house' by specialized machine-tools building companies, and it is highly customized by the builder. On the other hand, the CNC equipment is added as a separate module. This module is provided by a small number of developers and there are only a limited number of such solutions on the market. By analogy, machine-tools structures could be seen as a computer (manufactured by many companies), while the CNC equipment could be seen as a CPU unit (microprocessor) equipping that computer, which is manufactured by a significantly smaller number of companies.

When comparisons between $\mathrm{CNC}$ machine-tools are considered, one has to take into consideration the both the performances of the machine-tool and the ones of the CNC equipment. Thus, there appear two different issues:

- Machine-tools characteristics are measurable, but in order to assess them, the user needs access to the physical system, or to its documentation (provided as technical and commercial offer by the machine-tools building company);

- $\quad$ CNC equipment performances are non-measurable, but due the fact that CNC equipment solutions are widely available on many CNC machine-tools already in operations, the existing previous experience and information could be valorized for comparison.

The rapid development of the machine-tools systems witnessed in the last years, corroborated with the latest advancements in the computers and communication technology lead to wide spreading of $\mathrm{CNC}$ multi-axis working centers in all branches of manufacturing industry. At present, one cannot 
consider selecting between machine-tools alternatives, without considering the $\mathrm{CNC}$ equipment as one of the most important features. However, it is quite hard to assess the performances of a $\mathrm{CNC}$ equipment in order to make a selection. On the other hand, the other technical features of a machine-tool system also have to be considered when the final goal is to select the best solution.

The proposed approach is based upon a combined AHP and fuzzy method, using seven technical criteria for comparing the alternatives. Four criteria were quantitative ones and can be expressed using crisp values, while the other three (related with the performance of the CNC equipment) were qualitative ones and had to be expressed by processing vague data, collected by means of questionnaires, using fuzzy techniques.

The main goal of the proposed approach was to develop a simple yet effective method to compare different alternatives of $\mathrm{CNC}$ machine-tools. In order to fulfil this goal, the following objectives were stated:

- Establishing a list of quantitative criteria to assess the performances of the considered alternatives of machine-tools (by accessing their technical documentations);

- Establishing a list of qualitative criteria to assess the performance of the CNC equipment equipping the considered alternatives, by valorizing the existing experience with this equipment;

- Developing and running an AHP taking into consideration both quantitative and qualitative criteria;

- Developing a fuzzy system to process the qualitative criteria in order to extract crisp values for AHP;

- $\quad$ Testing the robustness of the results of the AHP by unfolding a sensitivity analysis.

\section{Materials and Methods}

\subsection{Methodology}

The entire process of CNC machine-tools selection was unfolded in a big manufacturing company (working in the field of automotive industry), which intended to upgrade its inventory of machining systems by purchasing a new type of five-axis CNC machining center. Four alternatives of five-axis $\mathrm{CNC}$ machining centers were considered for analysis. The comparison between alternatives targeted both the performances of the machine-tools and the performances of the CNC equipment fitted on every machine. Each considered machining center was manufactured by a different company. The CNC equipment on every considered machines-tool were well-known and widely spread solutions. The users of the manufacturing company were familiar with the $\mathrm{CNC}$ solutions considered, while the actual machine tools represented for them new and upgraded machining systems.

The main goal of the proposed approach was to select between four alternatives of CNC machine-tools existing on the market.

The first step of the approach was to define a set of criteria for comparison. The criteria were divided into quantitative ones $(\mathrm{C} 1, \mathrm{C} 2, \mathrm{C} 3$, and $\mathrm{C} 4)$, related to the machine-tools, and qualitative ones (C5, C6, and C7), related to the CNC equipment.

The second step involved a pairwise comparison of the seven criteria (C1-C7), as a first stage of AHP. The comparison was based upon the expertise of the authors and other specialists in the field.

The third step involved the build of the fuzzy systems used to express the qualitative criteria C5, C6, and C7 by crisp values, needed for the next stages of AHP.

The fourth step was dedicated to collect the data by means of questionnaires. The data collection made use by the previous experience existing in the manufacturing company related to the $\mathrm{CNC}$ equipment. Because the considered CNC equipment is wide-spread and well-known, the data collection process was not dependent of the physical availability of the four CNC machine-tools considered. The data collected was processed by the fuzzy systems which provided as outputs crisp values for $\mathrm{C} 5, \mathrm{C} 6$, and $\mathrm{C} 7$ criteria, which could be further used for the second stage of the AHP. 
During the fifth step, the second stage of the AHP was unfolded, which required the evaluation of the four alternatives of $\mathrm{CNC}$ machine-tools with regards of the proposed criteria. As result, the most beneficial alternative was selected.

The sixth step was dedicated to an evolution of the robustness of the results of the AHP, by running a sensitivity analysis.

A flowchart of the proposed approach is presented in Figure 2.

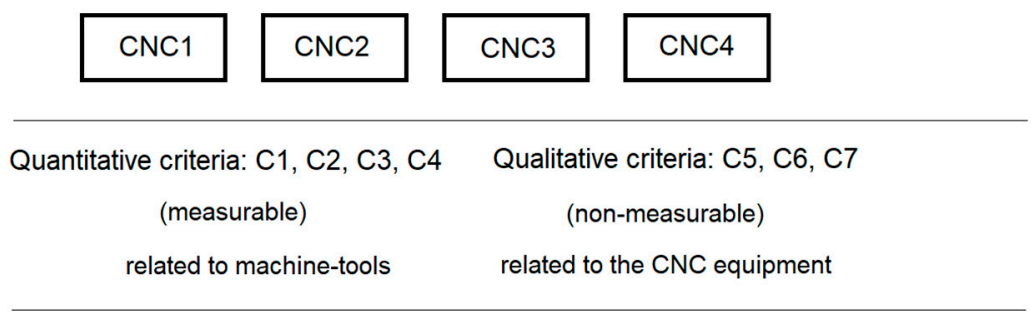

Performing the pairwise comparisons of $\mathrm{C} 1, \mathrm{C} 2, \mathrm{C} 3, \mathrm{C4}, \mathrm{C5}, \mathrm{C6}, \mathrm{C7}$ criteria

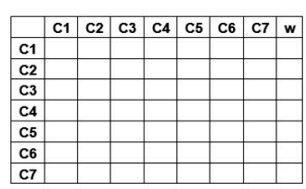

Building the fuzzy system for expressing the $C 5, C 6, C 7$ by crisp values Introducing sub-criteria

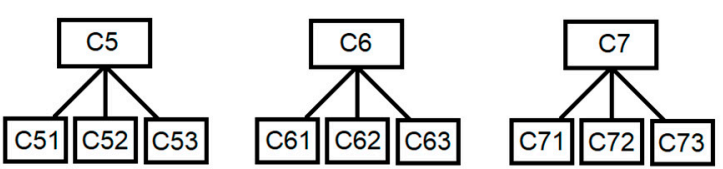

Collecting data by questionnaires and processing them by the fuzzy system C5, C6, and C7 criteria are related to CNC equipment which are wide-spread ad well-known (data collection is not dependent on the physical availability of the four alternatives)

Evaluation of the alternatives with regards of the proposed criteria

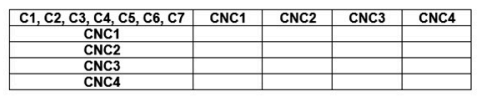

Testing the robustness of the results

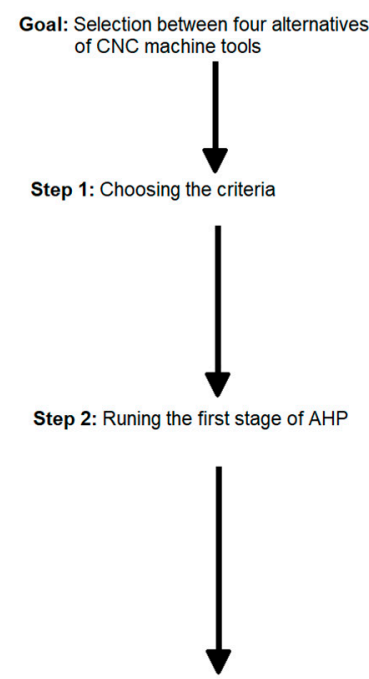

Step 3: Building the fuzzy system

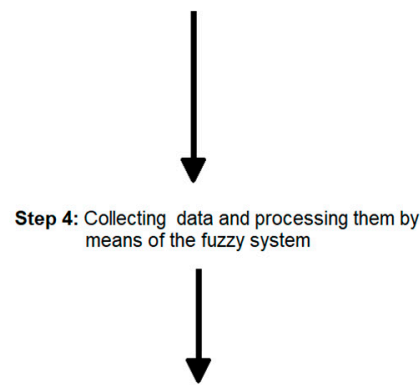

Step 5: Running the second stage of AHP

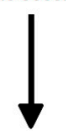

Step 6: Running the senzitivity analysis

Figure 2. Flowchart of the proposed approach.

\subsection{Criteria}

The proposed method is relying on a AH approach combined with fuzzy inference systems to quantify some criteria.

To select between four alternatives of five-axis machining centers, seven criteria were proposed: C1 -traverse speed, C2 - thrust, C3—spindle power, C4—spindle speed, C5-programming flexibility, C6-operation easiness, and C7-setup time. The significance of each criterion is presented below:

- Traverse speed (C1), measured in meters / minute $(\mathrm{m} / \mathrm{min})$ is the maximum positioning velocity which can be achieved on translational axes of the CNC machine-tool (X, Y, Z) during no-load regime (no cutting occurs during this regime). This velocity expresses the ability of the machine slides to change the position between the phases of a cutting operation, to approach and plunge to a new cutting position. Traverse speed is an important measure of the productivity of the machine-tools. It is considered that the time spent by the machine slides to reposition between 
the cutting phases has a significant influence upon the overall machining time. A higher traverse speed will reduce this time and consequently will increase the productivity, thus reducing the costs and increasing the economic efficiency of the production. Nowadays, some machine-tools manufacturers try to reduce the prices by lowering the achievable traverse speed (a lower traverse speed requires cheaper guiding systems on the machine-tool). This approach is associated with the risks of lowering the machine efficiency and reducing the productivity of the machining process.

- Thrust (C2), measured in Newtons (N), represents the maximum feed force which can be taken over by the machine slides on the translational axes $(X, Y, Z)$ during the cutting regime. This force expresses the ability of the machine-tools to cut hard materials, such as highly alloyed steels, reported at the level of the feed drives. The allowable thrust also has a significant influence upon the cutting depth, in a proportional way. Cutting depth (the amount of material which can be removed by the cutting tool on a single pass) is also a measure of the productivity of the cutting operation.

- $\quad$ Spindle power (C3), measured in kilowatts (KW), represents the power available at the main spindle of the machine-tool and together with the thrust characterizes the ability of the equipment to machine hard materials, by this time reported at the main drive level. In order to machine hard materials (mild steel or alloyed steel) the spindle power should exceed at least $1 \mathrm{KW}$. The size of the spindle power also influences the cutting depth, this time at the main spindle level.

- Spindle speed (C4), measured in revolutions/minutes (rev/min), represents the maximum speed achievable by the main spindle and consequently the maximum speed of the milling tool. It determines the maximum cutting speed and is also an important measure of the productivity of the machine-tool. The spindle speed is an indirect expression of the cutting speed. The higher the cutting speed is, the higher the material removal rate, which is also an important factor with regards of the productivity.

- CNC machine-tools are working in an automatic way, under the control of a computer program, called CNC code. This program has to be made by the programmers using specialized software. Some CNC equipment is fitted with additional tools which allow the user to interactively build the $\mathrm{CNC}$ code on the machine, or to simulate the machining process. The $\mathrm{C} 5$ criterion (programming flexibility) aims to assess these additional capabilities of the CNC equipment.

- $\quad \mathrm{CNC}$ machine tools are complex systems and have to be operated by trained personnel. It is important that the operating process to be a simple and straightforward one, while also allowing an efficient control of all operating phases (such as manual moves of the machine slides). It is also important that the input/output of the NC code and its editing to be made in an effective way. The $\mathrm{C} 6$ criterion (operation easiness) aims to assess these characteristics.

- As any complex system, the CNC machine-tools require a significant amount of setup. These include setting up the origin of the part, measuring the tools offsets and fixing/unfixing the workpiece on the machine. Setup time is also one of the most important factors which influences the productivity (the longer setup time is, the longer the machine-tools does not cut and consequently parts are not machined). On different machines, the setup phases are performed differently (simpler/faster of complex/slower), facts which will be assessed by C7 criterion (setup time).

The first four parameters ( $\mathrm{C} 1-\mathrm{C} 4)$ are quantitative ones and can be expressed by crisp values, delivered by the machine manufacturer within the datasheet. The last three parameters $(C 5-C 7)$ are qualitative ones and consequently they are hard to link to numerical values.

\subsection{AHP Approach-Initial Stage}

The selection between the four five-axis CNC machining centers, the AHP method introduced by Saaty $[7,8]$ will be used. The approach is based on pairwise comparisons by comparing elements $i$ 
and $j$. As a result, the value $a_{i j}$ will be obtained. A given hierarchization criteria is used for making the comparison

$$
\begin{gathered}
a_{i j}=1 \text { for } i=j, \text { where } i, j=1,2, \ldots, n \\
a_{i j}=\frac{1}{a_{j i}} \text { for } i \neq j
\end{gathered}
$$

In this work, the judgement scale proposed by Saaty was considered for the pairwise comparisons: 1-equally important; 3-weakly more important; 5-strongly more important; 7-demonstrably more important; 9 -absolutely more important. The values in between $(2,4,6$, and 8$)$ represent compromise judgements.

Each of the seven criteria proposed for comparing the five-axis machining centers were compared pairwise against each other. The comparison results were synthesized in the preference matrix A from Table 1.

Table 1. Preference matrix A.

\begin{tabular}{cccccccc}
\hline & C1 & C2 & C3 & C4 & C5 & C6 & C7 \\
\hline C1 & 1 & $1 / 3$ & $1 / 7$ & $1 / 3$ & $1 / 5$ & $1 / 9$ & $1 / 7$ \\
C2 & 3 & 1 & $1 / 3$ & $1 / 3$ & $1 / 3$ & $1 / 5$ & $1 / 3$ \\
C3 & 7 & 3 & 1 & 5 & 3 & 3 & 3 \\
C4 & 3 & 3 & $1 / 5$ & 1 & $1 / 3$ & $1 / 3$ & $1 / 3$ \\
C5 & 5 & 3 & $1 / 3$ & 3 & 1 & $1 / 3$ & $1 / 3$ \\
C6 & 9 & 5 & $1 / 3$ & 3 & 3 & 1 & 3 \\
C7 & 7 & 3 & $1 / 3$ & 3 & 3 & $1 / 3$ & 1 \\
\hline
\end{tabular}

For clarity, it will be explained below how the first line of Table 1 was completed:

- Traverse speed (C1) is a measure of productivity, but is weakly more important than thrust (C2), which expresses the ability of machining hard materials (or using high cutting depths);

- $\quad$ Spindle power (C3) expresses in the highest degree the ability of the machine-tool to machine hard materials, so it is demonstrably more important that the traverse speed (C2);

- $\quad$ Spindle speed (C4) is also a measure of productivity, but it acts upon the cutting speed, so it can be considered weakly more important than traverse speed (C1);

- The ability to program the CNC machine-tool in a flexible way (C5) is considered strongly more important than the traverse speed (C1);

- Operating the CNC machine-tool with ease (C6) is one of its paramount features, so it is considered absolutely more important than the traverse speed (C1);

- Setup time (C7) is also an important measure of the productivity of a machining process, and it was considered demonstrably more important than the traverse speed (C1).

Following the AHP approach, the next step involves the normalization of the preference matrix A, by generating the matrix $\mathrm{B}$ according to

$$
\begin{aligned}
B & =\left[b_{i j}\right] \\
b_{i j} & =\frac{a_{i j}}{\sum_{i=1}^{n} a_{i j}}
\end{aligned}
$$

Table 2 presents the normalized matrix B. On the last column of matrix B, the eigenvector $\mathrm{w}$, calculated as the arithmetic averages from the row of the normalized comparison matrix, according to Equation (3), was placed.

$$
w_{i j}=\frac{\sum_{i=1}^{n} b_{i j}}{n}
$$

In order to test the consistency of the pairwise comparisons [7,8,37], the maximal eigenvalue has to be calculated

$$
\lambda_{\max }=\frac{1}{n} \sum_{i=1}^{n} \frac{(A w)_{i}}{w_{i}}=7.6420
$$


$\lambda_{\max }$ from Equation (4) represents the largest eigenvalue of the matrix [7].

Table 2. Normalized matrix B.

\begin{tabular}{ccccccccc}
\hline & C1 & C2 & C3 & C4 & C5 & C6 & C7 & w \\
\hline C1 & 0.0286 & 0.0182 & 0.0534 & 0.0213 & 0.0184 & 0.0209 & 0.0175 & 0.0255 \\
C2 & 0.0857 & 0.0545 & 0.1246 & 0.0213 & 0.0307 & 0.0377 & 0.0409 & 0.0565 \\
C3 & 0.2000 & 0.1636 & 0.3737 & 0.3192 & 0.2761 & 0.5649 & 0.3684 & 0.3237 \\
C4 & 0.0857 & 0.1636 & 0.0747 & 0.0638 & 0.0307 & 0.0628 & 0.0409 & 0.0746 \\
C5 & 0.1429 & 0.1636 & 0.1246 & 0.1915 & 0.0920 & 0.0628 & 0.0409 & 0.1169 \\
C6 & 0.2571 & 0.2727 & 0.1246 & 0.1915 & 0.2761 & 0.1883 & 0.3684 & 0.2398 \\
C7 & 0.2000 & 0.1636 & 0.1246 & 0.1915 & 0.2761 & 0.0628 & 0.1228 & 0.1630 \\
\hline
\end{tabular}

By using the random consistency index table (Table 3), which was introduced in [8], the consistency ratio $C R$ may be calculated (for a seven-dimensional matrix, the $r$ coefficient is 1.32).

Table 3. Values for CI indices.

\begin{tabular}{ccccccccccc}
\hline Size of Matrix (n) & $\mathbf{1}$ & $\mathbf{2}$ & $\mathbf{3}$ & $\mathbf{4}$ & $\mathbf{5}$ & $\mathbf{6}$ & $\mathbf{7}$ & $\mathbf{8}$ & $\mathbf{9}$ & $\mathbf{1 0}$ \\
\hline Random average CI (r) & 0 & 0 & 0.58 & 0.90 & 1.12 & 1.24 & 1.32 & 1.41 & 1.45 & 1.51 \\
\hline
\end{tabular}

According to the result presented in Equation (5), the value of CR was found to smaller than $10 \%$ $(8.1055 \%)$. This value certifies the fact that the pairwise comparisons which were made in the process of structuring the matrices $\mathrm{A}$ and $\mathrm{B}$ are consistent $[8,37]$.

$$
C R=\frac{\lambda_{\max }-n}{r(n-1)}=8.1055 \%
$$

\subsection{Fuzzy Inference Systems}

As stated before, the $\mathrm{C} 5-\mathrm{C} 7$ criteria are qualitative ones and hard to be quantified by numerical values. To overcome that, fuzzy inference systems were built, under Mathworks MATLAB software package for each criterion.

Programming flexibility of the five-axis CNC machine-tools (C5) is the first qualitative criterion taken into consideration. It is very hard to quantify its value, so a fuzzy inference system was proposed for that. The criterion was divided into three sub-criteria, which were considered as input for the fuzzy system:

- C51-interactive programming on the machine (the operator can access and use machine cycles). Usually, the CNC programs for machining complex parts are generated by means of CAD (computer aided design)/CAM (computer aided manufacturing) software packages. However, on modern $\mathrm{CNC}$ controllers, part programs and machining processes (for simple parts) can be created and customized by the operator, in an easy, intuitive way, without the need of knowing any CNC programming language, at the level of the operator panel of the machine tool;

- $\quad$ C52-realistic simulation as a measure of how much information is provided to the operator by the simulation engine available at the level of the CNC controller. Nowadays, almost any CNC equipment is capable to simulate the machining process, based upon the CNC code generated by means of CAM software. However, the performance level of these simulations is very different form a CNC controller to another. This criterion will evaluate if the operator panel allows the definition and simulation of the workpiece, if it simulates the material removing (rather than simulating only the toolpaths), if it simulates the movements of the machine slides and rotary tables and if collision warnings are issued in this stage; 
- C53-monitoring process parameters. This criterion will evaluate if technological forces, torques, speeds, and feeds within the process can be measured and visualized and if their values can be saved for off-line analysis.

The fuzzy inference system for C5 criterion, presented in Figure 3, has three inputs (C51, C52 and C53) and one output (C5). The software tool used for building the fuzzy system was Mathworks MATLAB R2016a, with Fuzzy Logic Toolbox, version 2.2.23.

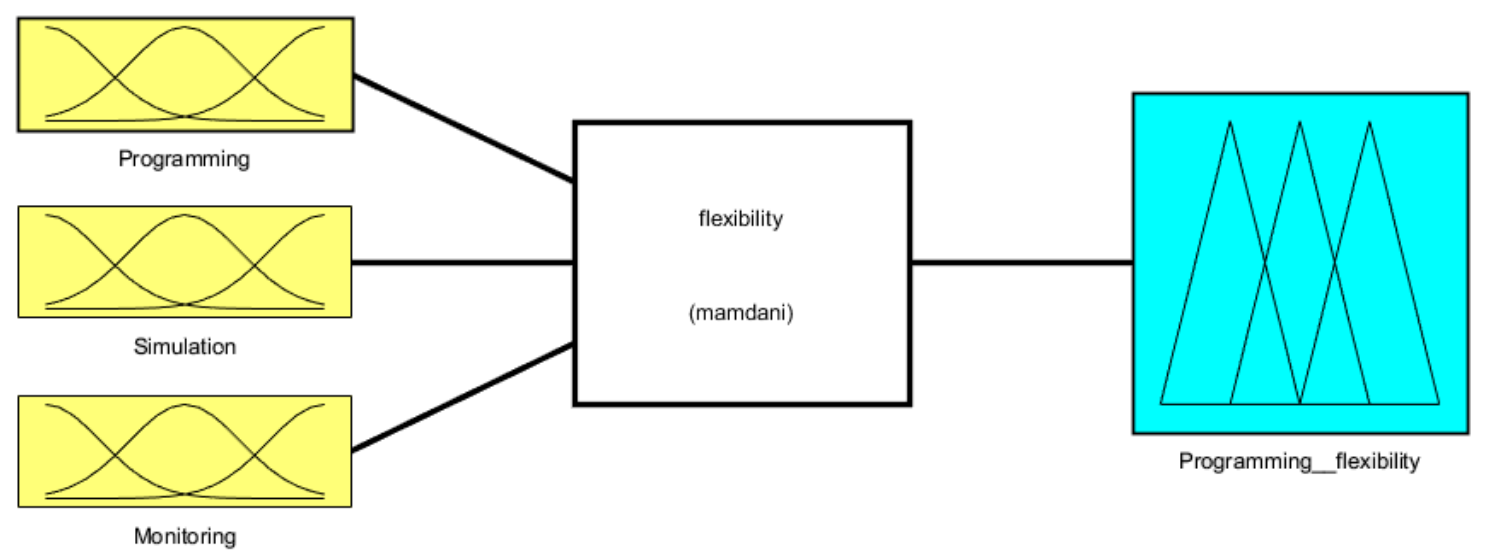

Figure 3. Fuzzy inference system for the 'programming flexibility' criterion.

The linguistic variables used and the linguistic degrees for fuzzifying the inputs and outputs are presented below:

- For C51 (interactive programming) input: $\mathrm{CP}$ —complex programming; $\mathrm{MP}$-medium complex programming; IP-intuitive programming.

- For C52 (realistic simulation) input: LR—low realistic; MR—-medium realistic; HR—high realistic.

- For C53 (monitoring process parameters) input: LM-limited monitoring; MM-medium monitoring; $\mathrm{CM}-$ comprehensive monitoring.

- $\quad$ For the output C5: LOW, MEDIUM, HIGH.

Triangular membership functions are used for both inputs and output. The range for all membership functions is $[0,10]$. The membership functions for input C51 is presented in Figure 4, while the membership function for output $\mathrm{C} 5$ is presented in Figure 5.

In order to clarify the concepts of linguistic variable and linguistic degree, let us consider the following, Given the linguistic variable 'C51-interactive programming', three linguistic degrees were defined $(\mathrm{CP}$-complex programming; $\mathrm{MP}$-medium complex programming; $\mathrm{IP}$-intuitive programming), each one associated with some typical membership functions, as presented in Figure 4. It can be seen that the membership function for the linguistic degree $\mathrm{CP}$-complex programming is a line, which indicates the fact that between 0 and 4 interactive programming is considered to be complex, with different levels of trust between 0 and 1 , the closer the deterministic value being to 0 , the higher its membership degree will be to the respective label. Similarly, for the linguistic degree $\mathrm{MP}$-medium complex programming, the maximal level of membership to this category is considered corresponding to the value of 5 . Values smaller or higher than this lead to the decrease of the trust level relatively to the attribute MP. The linguistic degree IP-intuitive programming is applied to the values higher than 6, with steadily higher trust levels as the value increases to 10 , and where programming is considered to be intuitive with a maximal trust level (equal to 1).

For the membership functions from Figure 4 we can state, for example, that programming with the value 2 is complex with a confidence level (the value of the membership function) of 0.50 , it is also medium complex with a confidence level of around 0.25 and it is intuitive with the confidence level zero. 


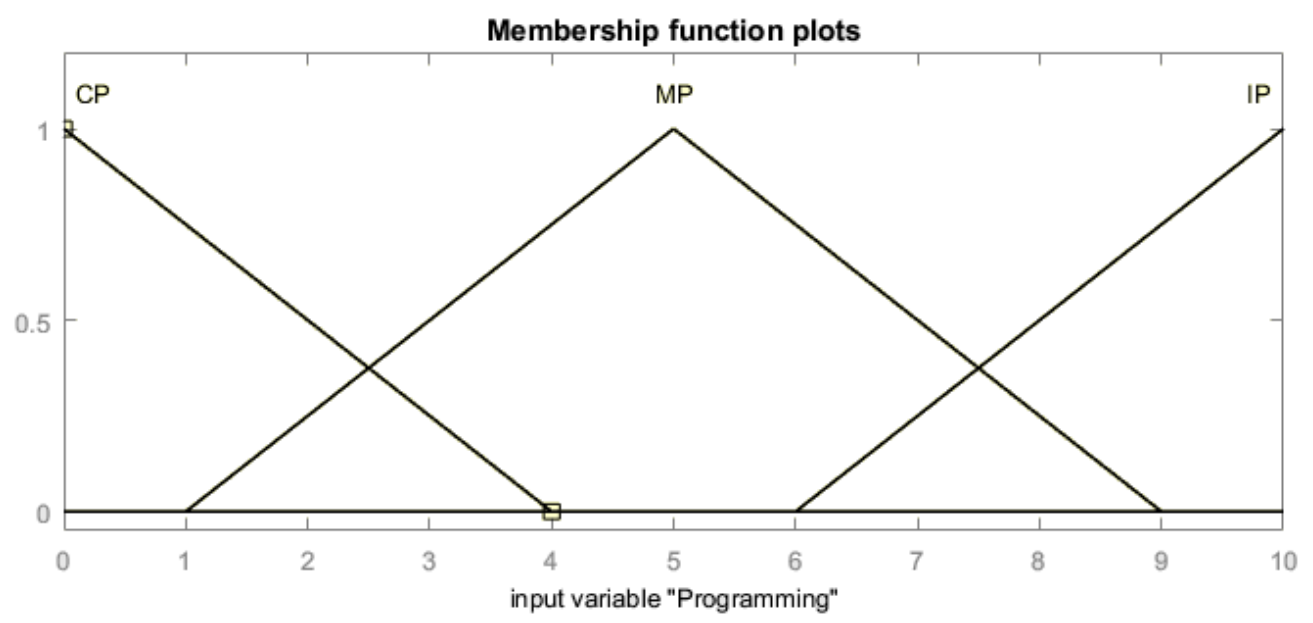

Figure 4. Membership function for the 'interactive programming' input.

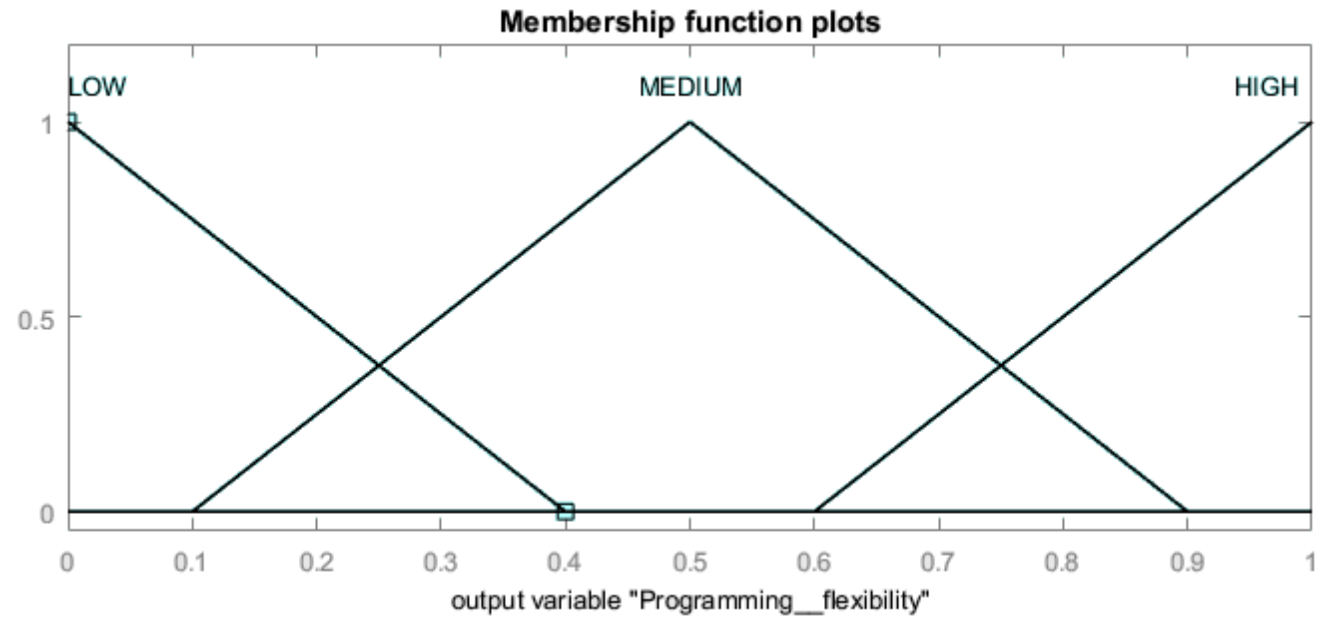

Figure 5. Membership function for the 'programming flexibility' output.

A synthetic presentation of the fuzzy rules for the 'programming flexibility' variable is presented in Table 4. All logical operators connecting the input variables are "AND" operators.

Table 4. Fuzzy rules for the 'programming flexibility' fuzzy inference system.

\begin{tabular}{cccc}
\hline $\begin{array}{c}\text { Interactive } \\
\text { Programming }\end{array}$ & Realistic Simulation & $\begin{array}{c}\text { Monitoring Process } \\
\text { Parameters }\end{array}$ & $\begin{array}{c}\text { Programming } \\
\text { Flexibility }\end{array}$ \\
\hline IP & MR & - & HIGH \\
- & HR & - & HIGH \\
MP & MR & CM & HIGH \\
MP & MR & MM & MEDIUM \\
CP & MR & CM & MEDIUM \\
- & MR & - & LOW \\
- & LR & LM & LOW \\
CP & MR & &
\end{tabular}

Operation easiness (C6) is the second qualitative criterion taken into consideration in this approach. A fuzzy inference system, with three inputs and one output was also build for this criterion. The considered inputs were the following:

- C61-ergonomics of the operator panel. This input assesses if the operator panel on the CNC equipment is easy to use, if the significant areas on the panel are well defined and the buttons within the panel have easy-to-identify function; 
- C62-control of the manual machine slide movements. This input assesses if the manual movements of the machine slides, in the jogging regime are easy to control, if there exist separate buttons for each manual movement, either linear or rotational;

- C63-editing of the program. This input assesses if the editing of the NC code is simple and straightforward, if the CNC equipment is able to identify syntax errors, if changes in the NC code can be made fast and easy.

The structure of fuzzy inference system for this criterion is presented in Figure 6.

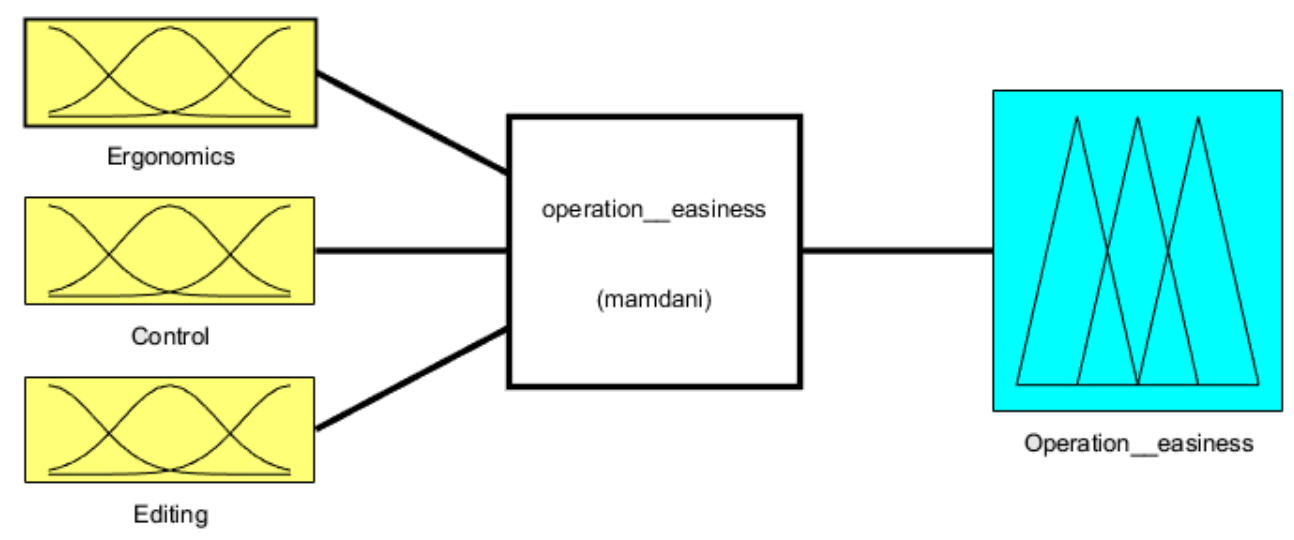

Figure 6. Fuzzy inference system for the 'operation easiness' criterion.

The linguistic variables and linguistic degrees used for fuzzifying the inputs and outputs are presented below:

- For C61 (ergonomics of the operator panel) input: LE-low ergonomics; ME-medium ergonomics; HE-high ergonomics.

- For C62 (control of the manual movements) input: CC—complex control; MC—medium control; EC-easy control.

- For C63 (editing of the program) input: CE-complex editing; ME-medium editing; EE- easy editing.

- $\quad$ For the output C6: LOW, MEDIUM, HIGH.

Triangular membership functions are used for both inputs and output for this fuzzy inference system also. The range for all membership functions is $[0,10]$. The membership functions for input C61 is presented in Figure 7, while the membership function for output C6 is presented in Figure 8.

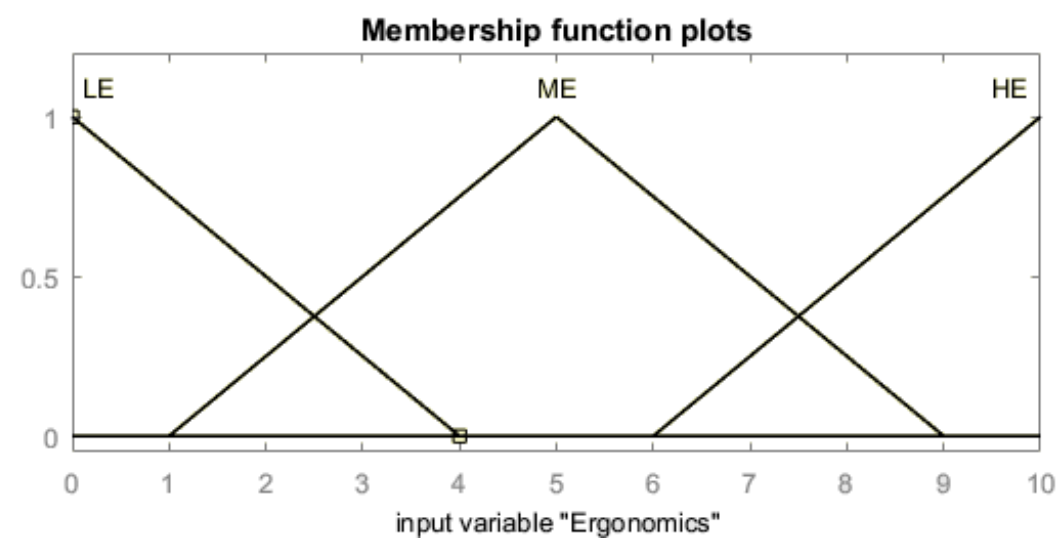

Figure 7. Membership function for the 'ergonomics of the operator panel' input. 


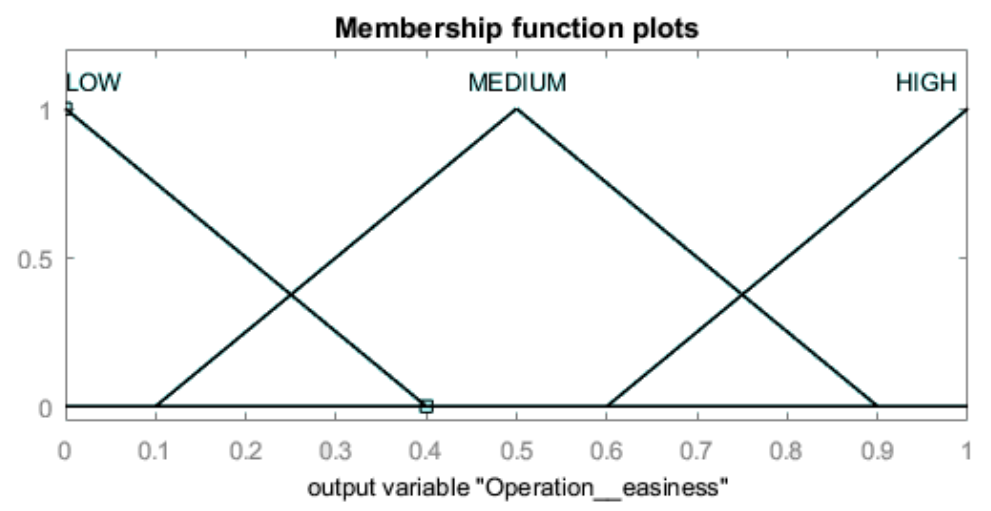

Figure 8. Membership function for the 'operation easiness' output.

A synthetic presentation of the fuzzy rules for the 'operation easiness' fuzzy inference system is presented in Table 5. All logical operators connecting the input variables are "AND" operators.

Table 5. Fuzzy rules for the 'operation easiness' fuzzy inference system.

\begin{tabular}{cccc}
\hline $\begin{array}{c}\text { Ergonomics of the } \\
\text { Operator Panel }\end{array}$ & $\begin{array}{c}\text { Control of the Manual } \\
\text { Movements }\end{array}$ & $\begin{array}{c}\text { Editing of the } \\
\text { Program }\end{array}$ & Operation Easiness \\
\hline LE & CC & - & LOW \\
- & CC & CE & LOW \\
ME & MC & - & MEDIUM \\
ME & EC & - & MEDIUM \\
HE & EC & EE & HIGH \\
ME & EC & & HIGH \\
\hline
\end{tabular}

Setup time (C7) is the third qualitative criterion taken into consideration in this approach. A fuzzy inference system, with three inputs and one output was also build for this criterion. The considered inputs were the following:

- $\quad$ C71 - setting up the origin of the part. This input assesses if the origin of the part can be set up fast an easy, and if the CNC equipment is fitted with automated cycles and devices specifically developed for this purpose;

- C72-measuring the tools offsets. This input assesses if the tools offsets can be measured on the machine in a fast, easy, and interactive way and if the CNC equipment has automated cycles for assisting the machine operator during this process;

- C73-fixing the workpiece. This input assesses if the process of fixing/unfixing the workpiece on the machine table can be made in a fast and easy way, and if the CNC equipment provides automated cycles or visual and/or sound aids to assist the machine operator during this process.

The structure of fuzzy inference system for this criterion is presented in Figure 9.

The linguistic variables and linguistic degrees used for fuzzifying the inputs and outputs are presented below:

- For C71 (setting up the origin of the part) input: SO-slow origin set-up; $\mathrm{MO}$-medium origin set-up; FO-fast origin set-up.

- For C72 (measuring the tools offsets) input: ST—-slow tool offsets measuring; MT—medium tool offsets measuring 1; FT-fast tool offsets measuring.

- For C73 (fixing the workpiece) input: SF-slow workpiece fixing; MF-medium workpiece fixing; $\mathrm{FF}$-fast workpiece fixing.

- For the output C7: LOW, MEDIUM, HIGH. 


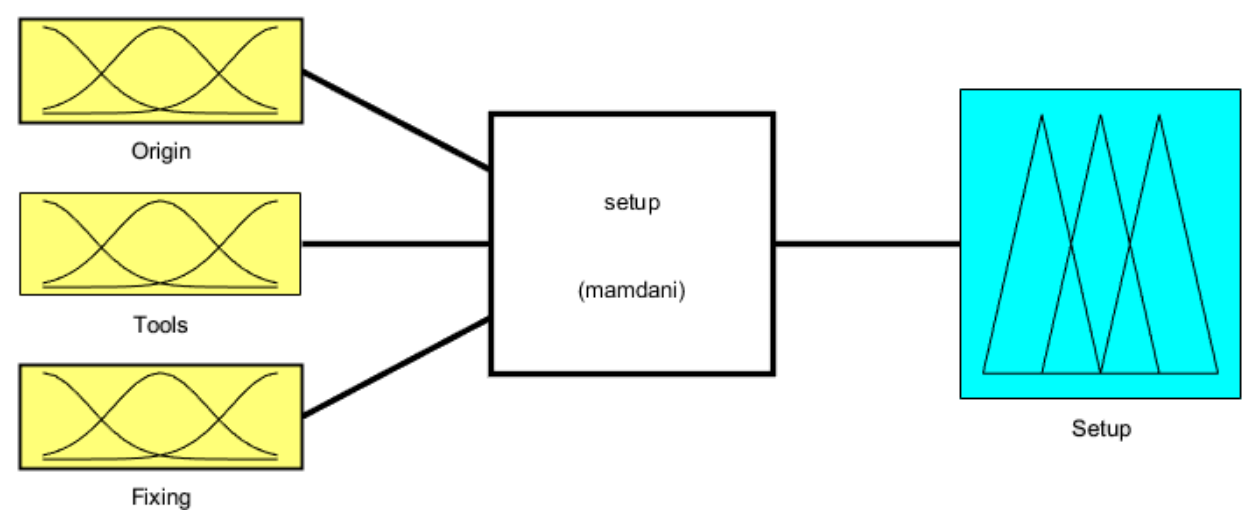

Figure 9. Fuzzy inference system for the 'setup time' criterion.

Triangular membership functions are used for both inputs and output for this fuzzy inference system also. The range for all membership functions is [0,10]. The membership functions for input C71 is presented in Figure 10, while the membership function for output is presented in Figure 11.

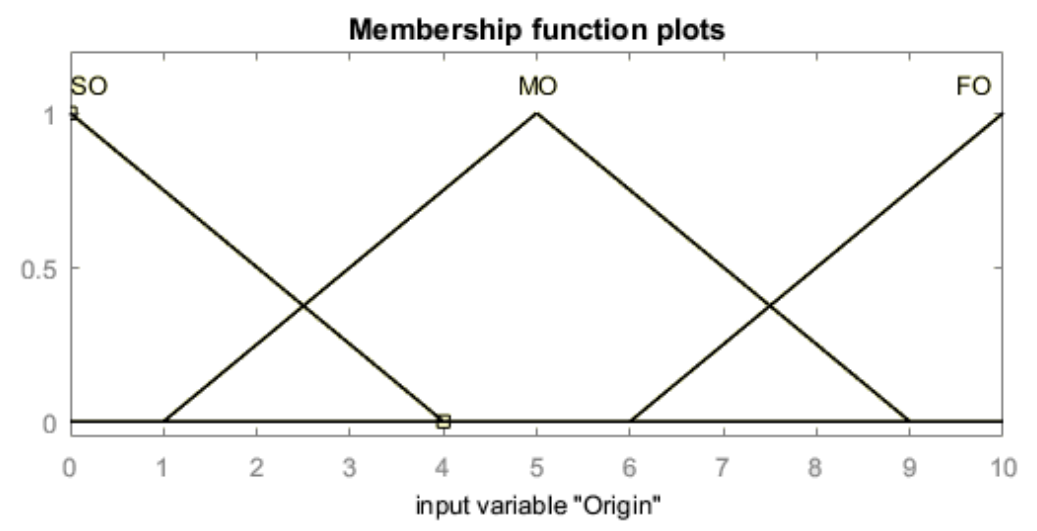

Figure 10. Membership function for the 'setting up the origin of the part' input.

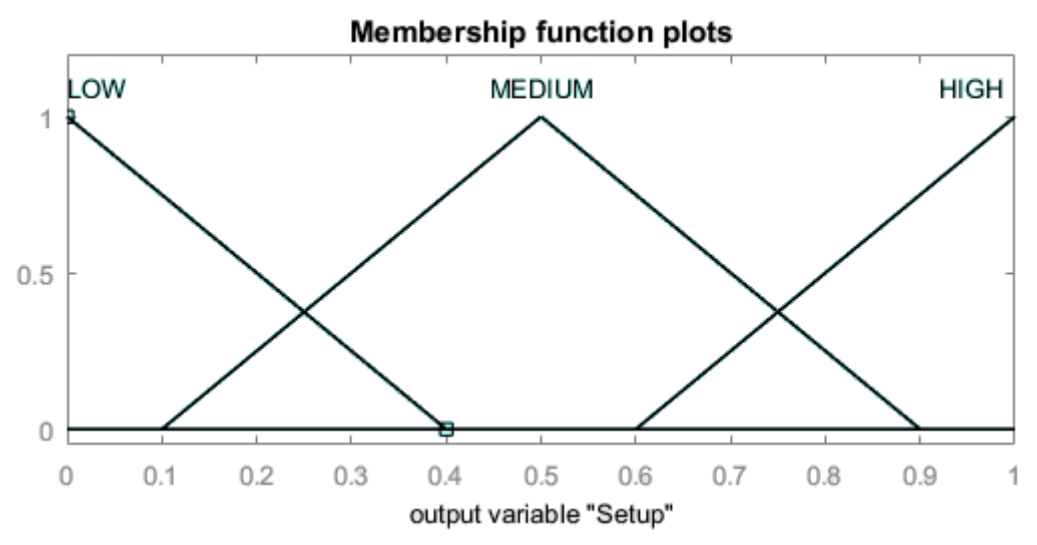

Figure 11. Membership function for the 'setup time' output.

A synthetic presentation of the fuzzy rules for the 'setup time' fuzzy inference system is presented in Table 6. All logical operators connecting the input variables are "AND" operators. 
Table 6. Fuzzy rules for the 'setup time' fuzzy inference system.

\begin{tabular}{cccc}
\hline $\begin{array}{c}\text { Setting Up the Origin } \\
\text { of the Part }\end{array}$ & Measuring the Tools Offsets & Fixing the Workpiece & Setup Time \\
\hline FO & MT & - & LOW \\
- & FT & - & LOW \\
MO & MT & FF & LOW \\
MO & MT & - & MEDIUM \\
SO & MT & FF & MEDIUM \\
- & MT & & MEDIUM \\
SO & ST & SF & HIGH \\
\hline
\end{tabular}

\section{Results}

The proposed method was used to select between four alternatives of five-axis machine-tools, which characteristics are presented in Table 7 . The data from Table 7 was extracted from the commercial brochure of each CNC machine-tool, which are publicly available for every potential customer.

Table 7. Characteristics of the four five-axis machine-tools.

\begin{tabular}{ccccc}
\hline Characteristic & CNC1 & CNC2 & CNC3 & CNC4 \\
\hline $\begin{array}{c}\text { Workspace X }(\mathrm{mm}) \times \mathrm{Y} \\
(\mathrm{mm}) \times \mathrm{Z}(\mathrm{mm})\end{array}$ & $762 \times 508 \times 508$ & $650 \times 520 \times 475$ & $762 \times 460 \times 460$ & $620 \times 520 \times 460$ \\
Traverse speed (m/min) & 30.5 & 42 & $40(\mathrm{X}, \mathrm{Y})$ & 36 \\
& $12,233(\mathrm{X}, \mathrm{Y})$ & 4800 & - & - \\
Thrust $(\mathrm{N})$ & $15,124(\mathrm{Z})$ & 21 & 11 & 11 \\
Spindle power $(\mathrm{KW})$ & 22.4 & 15,000 & 8000 & 12,000 \\
Spindle speed $(\mathrm{rev} / \mathrm{min})$ & 8100 & & & \\
\hline
\end{tabular}

Each of the four five-axis machine-tools was equipped with one of the main CNC equipment solutions existing on the market. Some of them were equipped with proprietary CNC solutions, while other ones were equipped with solutions provided by other well-known CNC equipment developers. However, every solution used by the four alternatives are well-known and widespread on the market.

For the quantitative criteria C1-C4, the data from Table 7 is enough for running the AHP process, but for the qualitative criteria C5-C7 supplementary data is needed. Mainly, the C5-C7 criteria are designed for the evaluation of the $\mathrm{CNC}$ equipment (the three main solutions existing on the market).

In order to gather the data for the proposed fuzzy inference systems (for C5-C7 criteria), a questionnaire was designed and distributed in a large machining company from Sibiu area. This factory runs more than 200 CNC machine-tools. As mentioned above, every CNC equipment used by the four analyzed alternatives are well-known and widespread on the market, so there were targeted in the questionnaires. Each item from the questionnaire had to be assessed the CNC equipment with a grade between 1-10, to allow its further fuzzification. The questionnaire used for gathering the data is in Appendix A.

A total number of 300 questionnaires were distributed (to machine tools operators, to technicians, and to engineers) and processed.

After feeding the fuzzy inference systems with the data from questionnaires, the results (expressed as crisp values for the qualitative criteria C5-C7) were processed and synthesized in Table 8.

Table 8. Crisp outputs of the fuzzy inference systems for the four alternatives (C5-C7 criteria).

\begin{tabular}{ccccc}
\hline Characteristic & CNC1 & CNC2 & CNC3 & CNC4 \\
\hline Programming flexibility & 7 & 9 & 10 & 8 \\
Operation easiness & 8 & 10 & 9 & 7 \\
Setup time & 8 & 10 & 9 & 7 \\
\hline
\end{tabular}


The next step of the AHP involves the evaluation of the alternatives, taking into consideration the proposed criteria. The evaluation for each one is presented in Tables 9-15. On the last column of each table the eigenvectors were introduced.

Table 9. Comparison of the four alternatives with regard to $\mathrm{C} 1$.

\begin{tabular}{cccccc}
\hline C1 & CNC1 & CNC2 & CNC3 & CNC4 & w \\
\hline CNC1 & 1 & $1 / 7$ & $1 / 5$ & $1 / 3$ & 0.0569 \\
CNC2 & 7 & 1 & 3 & 5 & 0.5579 \\
CNC3 & 5 & $1 / 3$ & 1 & 3 & 0.2633 \\
CNC4 & 3 & $1 / 5$ & $1 / 3$ & 1 & 0.1219 \\
\hline
\end{tabular}

Table 10. Comparison of the four alternatives with regard to $C 2$.

\begin{tabular}{cccccc}
\hline C2 & CNC1 & CNC2 & CNC3 & CNC4 & w \\
\hline CNC1 & 1 & 7 & 9 & 9 & 0.6727 \\
CNC2 & $1 / 7$ & 1 & 5 & 5 & 0.2122 \\
CNC3 & $1 / 9$ & $1 / 5$ & 1 & 1 & 0.0576 \\
CNC4 & $1 / 9$ & $1 / 5$ & 1 & 1 & 0.0576 \\
\hline
\end{tabular}

Table 11. Comparison of the four alternatives with regard to $C 3$.

\begin{tabular}{cccccc}
\hline C3 & CNC1 & CNC2 & CNC3 & CNC4 & w \\
\hline CNC1 & 1 & 3 & 9 & 9 & 0.5634 \\
CNC2 & $1 / 3$ & 1 & 9 & 9 & 0.3378 \\
CNC3 & $1 / 9$ & $1 / 9$ & 1 & 1 & 0.0494 \\
CNC4 & $1 / 9$ & $1 / 9$ & 1 & 1 & 0.0494 \\
\hline
\end{tabular}

Table 12. Comparison of the four alternatives with regard to $C 4$.

\begin{tabular}{cccccc}
\hline C4 & CNC1 & CNC2 & CNC3 & CNC4 & w \\
\hline CNC1 & 1 & $1 / 9$ & 3 & $1 / 7$ & 0.0771 \\
CNC2 & 9 & 1 & 9 & 5 & 0.6170 \\
CNC3 & $1 / 3$ & $1 / 9$ & 1 & $1 / 7$ & 0.0425 \\
CNC4 & 7 & $1 / 5$ & 7 & 1 & 0.2634 \\
\hline
\end{tabular}

Table 13. Comparison of the four alternatives with regard to $\mathrm{C} 5$.

\begin{tabular}{cccccc}
\hline C5 & CNC1 & CNC2 & CNC3 & CNC4 & w \\
\hline CNC1 & 1 & $1 / 5$ & $1 / 7$ & $1 / 3$ & 0.0569 \\
CNC2 & 5 & 1 & $1 / 3$ & 3 & 0.2633 \\
CNC3 & 7 & 3 & 1 & 5 & 0.5579 \\
CNC4 & 3 & $1 / 3$ & $1 / 5$ & 1 & 0.1219 \\
\hline
\end{tabular}

Table 14. Comparison of the four alternatives with regard to C6.

\begin{tabular}{cccccc}
\hline C6 & CNC1 & CNC2 & CNC3 & CNC4 & w \\
\hline CNC1 & 1 & $1 / 5$ & $1 / 3$ & 3 & 0.1219 \\
CNC2 & 5 & 1 & 3 & 7 & 0.5579 \\
CNC3 & 3 & 0.3333 & 1 & 5 & 0.2633 \\
CNC4 & $1 / 3$ & $1 / 7$ & $1 / 5$ & 1 & 0.0569 \\
\hline
\end{tabular}


Table 15. Comparison of the four alternatives with regard to C7.

\begin{tabular}{cccccc}
\hline C7 & CNC1 & CNC2 & CNC3 & CNC4 & w \\
\hline CNC1 & 1 & $1 / 5$ & $1 / 3$ & 3 & 0.1219 \\
CNC2 & 5 & 1 & 3 & 7 & 0.5579 \\
CNC3 & 3 & $1 / 3$ & 1 & 5 & 0.2633 \\
CNC4 & $1 / 3$ & $1 / 7$ & $1 / 5$ & 1 & 0.0569 \\
\hline
\end{tabular}

At this moment, using the data from Tables 9-14 the user can build matrix C. On the columns of matrix $C$ one has the eigenvectors resulting by comparing the four alternatives pairwise. The order of the columns within matrix $C$ takes into consideration the order of the criteria determined in Table 2: $\mathrm{C} 3, \mathrm{C} 6, \mathrm{C} 7, \mathrm{C} 5, \mathrm{C} 4, \mathrm{C} 2$, and $\mathrm{C} 1$. By performing the multiplication of matrix $\mathrm{C}$ and the vector $\mathrm{w}$, the preference vector $\mathrm{x}$ for the four analyzed alternatives is obtained.

The multiplication is presented in Equation (6).

Analyzing the results from (6), it can be stated that AHP method has designated CNC2 five-axis milling machine as the best alternative.

$$
x=C w=\left[\begin{array}{lllllll}
0.5634 & 0.1219 & 0.1219 & 0.0569 & 0.0771 & 0.6727 & 0.0569 \\
0.3378 & 0.5579 & 0.5579 & 0.2633 & 0.6170 & 0.2122 & 0.5579 \\
0.0494 & 0.2633 & 0.2633 & 0.5579 & 0.0425 & 0.0576 & 0.2633 \\
0.0494 & 0.0569 & 0.0569 & 0.1219 & 0.2634 & 0.0576 & 0.1219
\end{array}\right]\left[\begin{array}{l}
0.0255 \\
0.0565 \\
0.3237 \\
0.0746 \\
0.1169 \\
0.2398 \\
0.1630
\end{array}\right]=\left[\begin{array}{l}
0.2445 \\
0.4543 \\
0.2047 \\
0.0964
\end{array}\right]
$$

\subsection{Sensitivity Analysis}

In order to test the robustness of the results a sensitivity analysis method for the results of the AHP was suggested in [38]. The analysis is based upon the change of the weights, while maintaining the ranking order previously determined. This can be made by introducing a coefficient $\alpha \geq 0$ and transforming the matrix A into the matrix $\left[a_{i j}^{\alpha}\right]$. According to [32], if $\alpha>1$ more dispersed weights are obtained and if $\alpha \leq 1$ the weights become more concentrated, without affecting the ranking order previously determined.

Table 16 shows the weights values obtained for $\alpha=0.5,0.7,0.9,1.0,1.1,1.3,1.5$ (these values were proposed in [39]).

Table 16. Sensitivity analysis for the weights.

\begin{tabular}{cccccccc}
\hline \multicolumn{7}{c}{ Coefficient $\boldsymbol{\alpha}$} \\
\hline & $\mathbf{0 . 5}$ & $\mathbf{0 . 7}$ & $\mathbf{0 . 9}$ & $\mathbf{1 . 0}$ & $\mathbf{1 . 1}$ & $\mathbf{1 . 3}$ & $\mathbf{1 . 5}$ \\
\hline C1 & 0.0127 & 0.0178 & 0.0229 & 0.0255 & 0.0280 & 0.0331 & 0.0382 \\
C2 & 0.0282 & 0.0395 & 0.0508 & 0.0565 & 0.0621 & 0.0734 & 0.0847 \\
C3 & 0.1618 & 0.2266 & 0.2913 & 0.3237 & 0.3561 & 0.4208 & 0.4855 \\
C4 & 0.0373 & 0.0522 & 0.0672 & 0.0746 & 0.0821 & 0.0970 & 0.1119 \\
C5 & 0.0584 & 0.0818 & 0.1052 & 0.1169 & 0.1286 & 0.1520 & 0.1753 \\
C6 & 0.1199 & 0.1679 & 0.2158 & 0.2398 & 0.2638 & 0.3118 & 0.3597 \\
C7 & 0.0815 & 0.1141 & 0.1467 & 0.1630 & 0.1794 & 0.2120 & 0.2446 \\
\hline
\end{tabular}

Table 17 presents the simulation results of calculating the preference vector $\mathrm{x}$, for the weights from Table 16. 
Table 17. Results of the sensitivity analysis simulations for the preference vector $x$.

\begin{tabular}{|c|c|c|c|c|c|c|c|}
\hline \multicolumn{8}{|c|}{ Coefficient $\alpha$} \\
\hline & 0.5 & 0.7 & 0.9 & 1.0 & 1.1 & 1.3 & 1.5 \\
\hline \multicolumn{8}{|c|}{ Preference Vector $x$} \\
\hline CNC1 & 0.1223 & 0.1712 & 0.2201 & 0.2445 & 0.2690 & 0.3179 & 0.3668 \\
\hline $\mathrm{CNC2}$ & 0.2272 & 0.3180 & 0.4089 & 0.4543 & 0.4997 & 0.5906 & 0.6815 \\
\hline $\mathrm{CNC3}$ & 0.1024 & 0.1433 & 0.1842 & 0.2047 & 0.2252 & 0.2661 & 0.3071 \\
\hline $\mathrm{CNC} 4$ & 0.0482 & 0.0675 & 0.0868 & 0.0964 & 0.1061 & 0.1254 & 0.1447 \\
\hline
\end{tabular}

A graphical representation of the results of sensitivity analysis is presented in Figure 12. From the figure one may notice that the changes in the weights does not affect the order of the preference vectors $\mathrm{x}$. As a result, it can be stated that $\mathrm{CNC} 2$ is the best alternative for the entire range of the sensitivity analysis.

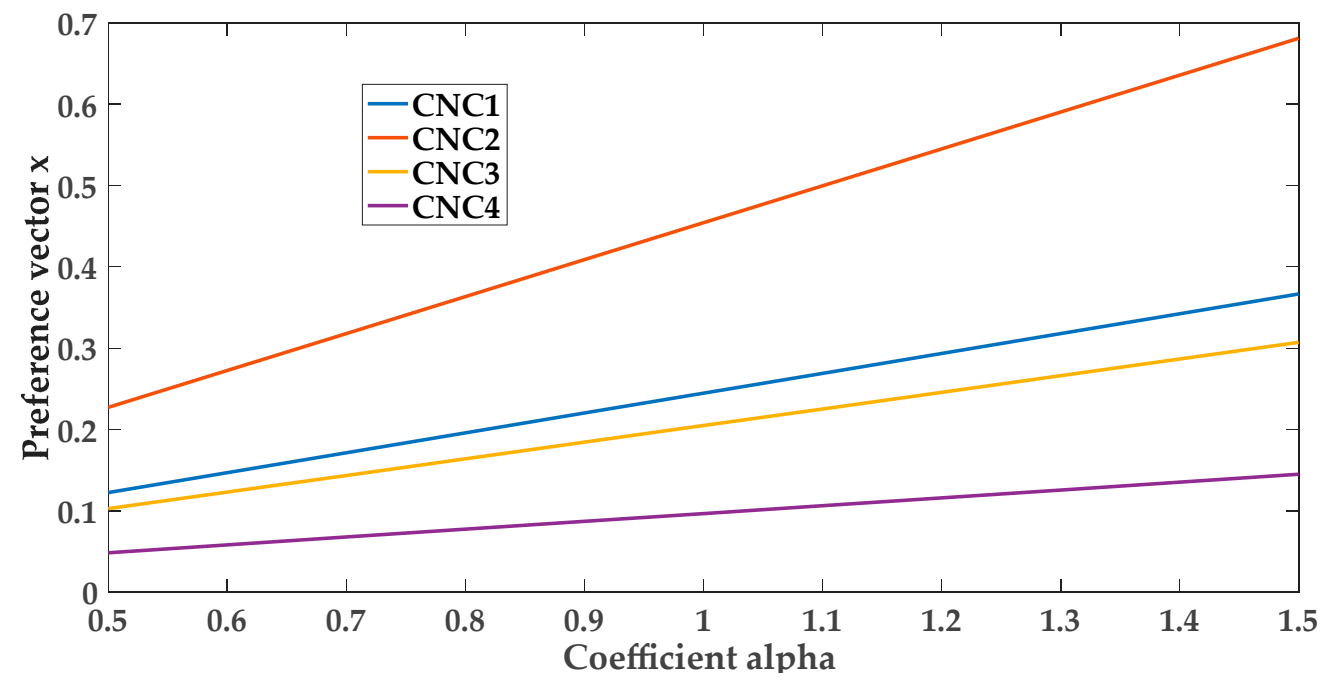

Figure 12. Sensitivity analysis-graphical representation.

\subsection{Conclusions}

On a very competitive market, machining processes must deliver accurate parts, just in time, in great numbers, at prices as lower as possible. These requirements are closely related with the CNC machine-tools overall performances, on which these processes are unfolded. Consequently, selecting the proper $\mathrm{CNC}$ machine-tools out of wide range of alternatives is a very important process, both from a managerial and technical point of view. It is difficult for managers and engineers to find a best compromise between these requirements. For example, machining accuracy (which is favored by engineers) is hardly achievable when great numbers and lower prices are also targeted. On the other hand, not fulfilling all these requirements by finding the best compromise (using the most appropriate $\mathrm{CNC}$ machine-tool) could inflict a great deal of both economic and technical risks upon the machining company.

The proposed approach takes into consideration not only the measurable characteristics of the machine-tools, but also the subjective, non-measurable features of the CNC equipment within the machines. The method integrates all these aspects and quantifies them into a straightforward approach, which can be implemented at factory level. The method requires technical expertise of engineers and technicians (available at factory level) for running the AHP and fuzzy systems. The data required for implementation are also easy to gather, by means of technical brochures (measurable data) and questionnaires (subjective data). A graphical synthesis of the conclusion is presented in Figure 13. 


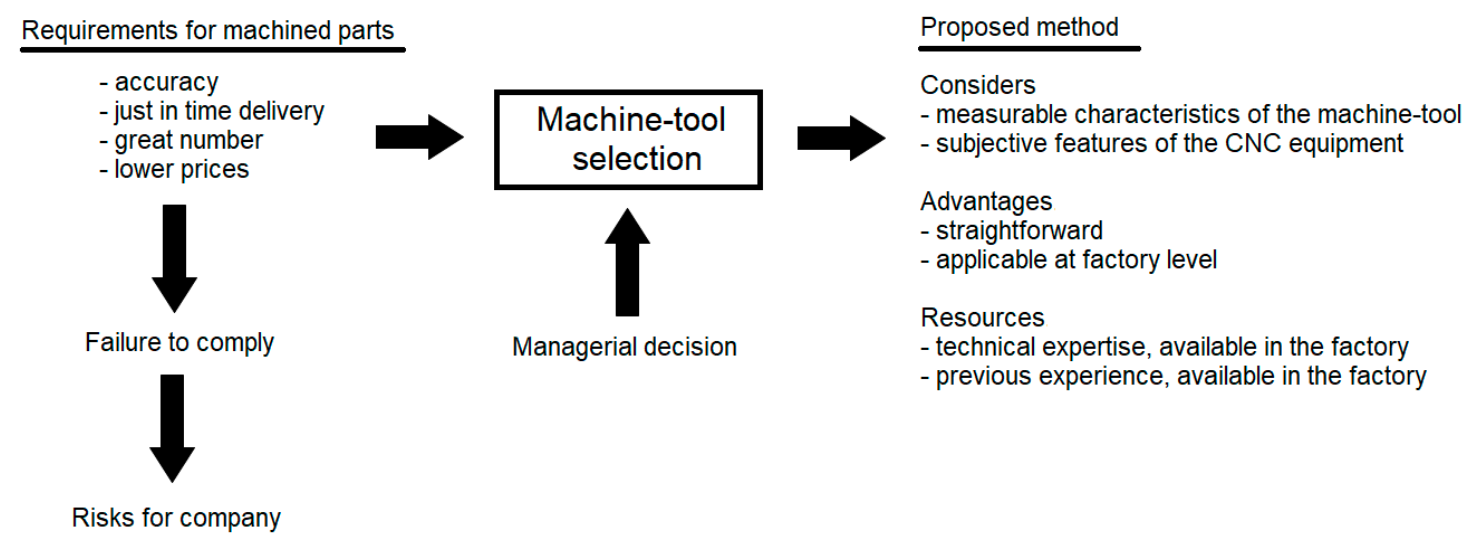

Figure 13. Graphical synthesis of the conclusion.

\section{Discussion}

Most of the approaches related with machine-tools selection do not take into consideration the fact that nowadays these systems are fitted with CNC. Thus, it was considered important to take this fact into consideration when selecting between alternative machine-tools. On the other hand, it is quite difficult to compare the performances of different $\mathrm{CNCs}$, due to two main reasons: first, there are several solutions available on the market and second, there are not quantitative parameters defined for unfolding this comparison. With regards of the first issue, the four analyzed alternatives were equipped with well-known and widespread CNC solutions. For the second issue, a fuzzy approach was proposed, for extracting crisp values from qualitative criteria, which were proposed for comparing the three above-mentioned CNC solutions.

Summarizing, the selection method took into consideration four alternatives of five-axis CNC machine-tools. The alternatives were compared by mean of the quantitative and qualitative parameters. Because the comparison is multi-criteria one, the AHP method was chosen as comparison tool, aided by three fuzzy inference systems.

The fuzzy systems were built to deal with vague data, related with the qualitative criteria. Taking into consideration the notoriety of the CNC solutions, questionnaires were used to gather the information processed by the fuzzy systems. The questionnaires were distributed in a large manufacturing company (which already uses all of the CNC equipment targeted) and the opinions of $\mathrm{CNC}$ operators, CNC technicians, and CNC programmers were consulted and quantized. In order to facilitate the fuzzifying process of the inputs, rather than asking them to evaluate the inputs with linguistic variables, they were required to grade each input with grades between 1 and 10, according to their 'feelings' and 'experiences'.

Finally, combining the crisp values obtained by using quantitative criteria, with the crisp values generated by the fuzzy systems, the AHP was unfolded, and the recommended alternative chosen. In order to certify the robustness of the AHP process, a sensitivity analysis was unfolded.

The results of this approach could be hardly influenced by the number of the processed questionnaires. Further research will be unfolded to study this influence.

Machine-tools selection process and the risks involved in it could be also related with preventive maintenance and reliability risks. There are some promising approaches reported in the literature with regards of industrial applications of reliability issues [40-43] and future studies will also tackle the links between machine-tools selection and reliability. Also, some validation methods for the proposed approach (other than sensitivity analysis) will be targeted in the future.

Author Contributions: Conceptualization, L.I.C., R.E.B., and S.G.R.; Methodology, R.E.B. and L.I.C.; Investigation R.E.B., S.G.R., and L.I.C.; Resources, L.I.C. and S.G.R.; Writing-original draft preparation, R.E.B.; Writing-review and editing, R.E.B.; Project administration, L.I.C.

Funding: This research was funded by Lucian Blaga University of Sibiu by means of the research grant LBUS-IRG-2017-03 and by Romanian Ministry of Research and Innovation CCCDI-UEFISCDI, by means of 
the project PN-III-P1-1.2-PCCDI-2017-0446/nr. 82PCCDI/2018, within PNCDI III, project title: “Intelligent manufacturing technologies for advanced production of parts".

Conflicts of Interest: The authors declare no conflict of interest.

\section{Appendix A. Questionnaire [Type of CNC Equipment}

Appendix A.1. Programming Flexibility

1.1. The CNC equipment allows fast and easy programming of simple machining cycles, in a graphical interactive way (drilling operations, pocket milling operations, etc.) directly on the machine, without the need of using ISO or TNC programming language. Editing these machining cycles is simple and intuitive, the parameters of the cycles are easy identifiable and understandable.

\begin{tabular}{llllllllll}
\hline 1 & 2 & 3 & 4 & 5 & 6 & 7 & 8 & 9 & 10 \\
\hline
\end{tabular}

1.2. The CNC equipment allows realistic simulation of the part programs on the machine, before running them. The tools/part movements are simulated, the workpiece is visualized and the material removing is depicted. The simulation also presents the movements of the machine slides and rotating tables and all the collisions (between tools and part and between machine slides and tables) are identified and signaled.

\begin{tabular}{llllllllll}
\hline 1 & 2 & 3 & 4 & 5 & 6 & 7 & 8 & 9 & 10 \\
\hline
\end{tabular}

1.3. The CNC equipment displays on the operator panel display specific values during the machining process (speeds, feeds, technological forces and torques, voltages and currents). These values can also be saved and transferred to another PC for further analysis.

\begin{tabular}{llllllllll}
\hline 1 & 2 & 3 & 4 & 5 & 6 & 7 & 8 & 9 & 10 \\
\hline
\end{tabular}

\section{Appendix A.2. Operation Easiness}

2.1. The operator panel is easy to use, the working areas on it are easy-to-identify and well defined, the buttons have easy-to-identify functions.

\begin{tabular}{llllllllll}
\hline 1 & 2 & 3 & 4 & 5 & 6 & 7 & 8 & 9 & 10 \\
\hline
\end{tabular}

2.2. Manual movements of the machine slides and tables during jogging regime are easy to control, there are separate buttons for each movement (axis), either linear or rotational.

\begin{tabular}{llllllllll}
\hline 1 & 2 & 3 & 4 & 5 & 6 & 7 & 8 & 9 & 10 \\
\hline
\end{tabular}

2.3. Editing of the NC code is simple and straightforward, and the CNC equipment is able to identify syntax errors, and changes in the NC code can be made fast and easy. As example of syntax errors, you may consider the following examples: programming the spindle rotation without setting up a speed, programming circular interpolation, but the final point is not lying on circle, etc.).

\begin{tabular}{lllllllllll}
\hline 1 & 2 & 3 & 4 & 5 & 6 & 7 & 8 & 9 & 10 \\
\hline
\end{tabular}


Appendix A.3. Setup Time

3.1. Setting up the origin of the part is made easy and fast, and the CNC equipment is fitted with automated cycles and devices specifically developed for this purpose.

\begin{tabular}{llllllllll}
\hline 1 & 2 & 3 & 4 & 5 & 6 & 7 & 8 & 9 & 10 \\
\hline & & & & & & & & & \\
\hline
\end{tabular}

3.2. Tools offsets can be measured on the machine fast, easy and interactive way and the CNC equipment has automated cycles for assisting the machine operator during this process.

\begin{tabular}{llllllllll}
1 & 2 & 3 & 4 & 5 & 6 & 7 & 8 & 9 & 10 \\
\hline
\end{tabular}

3.3. The process of fixing/unfixing the workpiece on the machine table can be made in a fast and easy way, and the CNC equipment provides automated cycles or visual and/or sound aids to assist the machine operator during this process.

\begin{tabular}{llllllllll}
\hline 1 & 2 & 3 & 4 & 5 & 6 & 7 & 8 & 9 & 10 \\
\hline
\end{tabular}

\section{References}

1. Kou, G.; Ergu, D.; Lin, C.; Chen, Y. Pairwise comparison matrix in multiple criteria decision making. Technol. Econ. Dev. Econ. 2016, 22, 738-765. [CrossRef]

2. Gbededo, M.A.; Liyanage, K. Identification and Alignment of the Social Aspects of Sustainable Manufacturing with the Theory of Motivation. Sustainability 2018, 10, 852. [CrossRef]

3. Kluczek, A. Quick Green Scan: A Methodology for Improving Green Performance in Terms of Manufacturing Processes. Sustainability 2017, 9, 88. [CrossRef]

4. Kulak, O.; Durmușoğlu, M.B.; Kahraman, C. Fuzzy multi-attribute equipment selection based on information axiom. J. Mater. Process. Technol. 2005, 169, 337-345. [CrossRef]

5. Kulak, O. A decision support system for fuzzy multi-attribute selection of material handling equipments. Expert Syst. Appl. 2005, 29, 310-319. [CrossRef]

6. Kulak, O.; Kahraman, C. Multi-attribute comparison of advanced manufacturing systems using fuzzy vs. crisp axiomatic design approach. Int. J. Prod. Econ. 2005, 95, 415-424. [CrossRef]

7. Saaty, T.L. The Analytic Hierarchy Process: Planning, Priority Setting, Resource Allocation; McGraw-Hill: New York, NY, USA, 1980; p. 287.

8. Saaty, T.L. Decision Making for Leaders: The Analytic Hierarchy Process for Decisions in a Complex Word; RWS Publication: Pittsburgh, PA, USA, 1990.

9. Garg, H.; Kumar, K. A novel exponential distance and its based TOPSIS method for interval-valued intuitionistic fuzzy sets using connection number of SPA theory. Artif. Intell. Rev. 2018, 1-30. [CrossRef]

10. Garg, H.; Kumar, K. An extended technique for order preference by similarity to ideal solution group decision-making method with linguistic interval-valued intuitionistic fuzzy information. J. Multi-Criteria Dec. Anal. 2008. [CrossRef]

11. Kumar, K.; Garg, H. Connection number of set pair analysis based TOPSIS method on intuitionistic fuzzy sets and their application to decision making. Appl. Intell. 2018, 48, 2112-2119. [CrossRef]

12. Kumar, K.; Garg, H. TOPSIS method based on the connection number of set pair analysis under interval-valued intuitionistic fuzzy set environment. Comput. Appl. Math. 2018, 37, 1319-1329. [CrossRef]

13. Garg, H. A new improved score function of an interval-valued Pythagorean fuzzy set based TOPSIS method. Int. J. Uncertain. Quantif. 2017, 7, 463-474. [CrossRef]

14. Garg, H.; Kaur, G. Extended TOPSIS method for multi-criteria group decision-making problems under cubic intuitionistic fuzzy environment. Sci. Iran. 2018. [CrossRef] 
15. Çimren, E.; Çatay, B.; Budak, E. Development of a machine tool selection system using AHP. Int. J. Adv. Manuf. Technol. 2007, 35, 363-376. [CrossRef]

16. Chakraborty, S.; Banik, D. Design of a material handling equipment selection model using analytic hierarchy process. Int. J. Adv. Manuf. Technol. 2006, 28, 1237-1245. [CrossRef]

17. Özdemir, R.G.; Ayağ, Z. An integrated approach to evaluating assembly-line design alternatives with equipment selection. Prod. Plan. Control 2010, 22, 194-206. [CrossRef]

18. Avram, O.; Stroud, I.; Xirouchakis, P. A multi-criteria decision method for sustainability assessment of the use phase of machine tool systems. Int. J. Adv. Manuf. Technol. 2011, 53, 811-828. [CrossRef]

19. Caputo, A.C.; Pelagagge, P.M.; Salini, P. AHP-based methodology for selecting safety devices of industrial machinery. Saf. Sci. 2013, 53, 202-218. [CrossRef]

20. Ayağ, Z.; Özdemir, R.G. A fuzzy AHP approach to evaluating machine tool alternatives. J. Intell. Manuf. 2006, 17, 179-190. [CrossRef]

21. Durán, O.; Aguilo, J. Computer-aided machine-tool selection based on a Fuzzy-AHP approach. Expert Syst. Appl. 2008, 34, 1787-1794. [CrossRef]

22. Dağdeviren, M. Decision making in equipment selection: An integrated approach with AHP and PROMETHEE. J. Intell. Manuf. 2008, 19, 397-406. [CrossRef]

23. Önüt, S.; Kara, S.S.; Efendigil, T. A hybrid fuzzy MCDM approach to machine tool selection. J. Intell. Manuf. 2008, 19, 443-453. [CrossRef]

24. Yurdakul, M.; Iç, Y.T. Analysis of the benefit generated by using fuzzy numbers in a TOPSIS model developed for machine tool selection problems. J. Mater. Process. Technol. 2009, 209, 310-317. [CrossRef]

25. Iç, Y.T.; Yurdakul, M. Development of a decision support system for machining center selection. Expert Syst. Appl. 2009, 36, 3505-3513. [CrossRef]

26. Kou, G.; Ergu, D. AHP/ANP theory and its application in technological and economic development: The 90th anniversary of Thomas L. Saaty. Technol. Econ. Dev. Econ. 2016, 22, 649-650. [CrossRef]

27. Ayağ, Z.; Özdemir, R.G. An intelligent approach to machine tool selection through fuzzy analytic network process. J. Intell. Manuf. 2011, 22, 163-177. [CrossRef]

28. Paramasivam, V.; Senthil, V.; Rajam Ramasamyn, N. Decision making in equipment selection: An integrated approach with digraph and matrix approach, AHP and ANP. Int. J. Adv. Manuf. Technol. 2011, 54, 1233-1244. [CrossRef]

29. Ayağ, Z.; Özdemir, R.G. Evaluating machine tool alternatives through modified TOPSIS and alpha-cut based fuzzy ANP. Int. J. Prod. Econ. 2012, 140, 630-636. [CrossRef]

30. Nguyen, H.-T.; Md Dawal, S.Z.; Nukman, Y.; Aoyama, H. A hybrid approach for fuzzy multi-attribute decision making in machine tool selection with consideration of the interactions of attributes. Expert Syst. Appl. 2014, 41, 3078-3090. [CrossRef]

31. Alberti, M.; Ciurana, J.; Rodríguez, C.A.; Özel, T. Design of a decision support system for machine tool selection based on machine characteristics and performance tests. J. Intell. Manuf. 2011, 22, 263-277. [CrossRef]

32. He, Y.; Li, Y.; Wu, T.; Sutherland, J.W. An energy-responsive optimization method for machine tool selection and operation sequence in flexible machining job shops. J. Clean. Prod. 2015, 87, 245-254. [CrossRef]

33. Kumar, R.; Garg, R.K. Optimal selection of robots by using distance based approach method. Robot. Comput. Integr. Manuf. 2010, 26, 500-506. [CrossRef]

34. Iç, Y.T.; Yurdakul, M.; Dengiz, B. Development of a decision support system for robot selection. Robot. Comput. Integr. Manuf. 2013, 29, 142-157. [CrossRef]

35. Liu, H.-C.; Ren, M.-L.; Wu, J.; Lin, Q.-L. An interval 2-tuple linguistic MCDM method for robot evaluation and selection. Int. J. Prod. Res. 2014, 52, 2867-2880. [CrossRef]

36. Parameshwaran, R.; Praveen Kumar, S.; Saravanakumar, K. An integrated fuzzy MCDM based approach for robot selection considering objective and subjective criteria. Appl. Soft Comput. 2015, 26, 31-41. [CrossRef]

37. Alonso, J.; Lamata, T.M. Consistency in the analytic hierarchy process: A new approach. Int. J. Uncertain. Fuzziness Knowl.-Based Syst. 2006, 14, 445-459. [CrossRef]

38. Hurley, W.J. The analytic hierarchy process: A note on an approach to sensitivity which preserves rank order. Comput. Oper. Res. 2001, 28, 185-188. [CrossRef]

39. Cabala, P. Using the analytic hierarchy process in evaluating decision alternatives. Oper. Res. Decis. 2010, 20, 5-23. 
40. Niwas, R.; Garg, H. An approach for analyzing the reliability and profit of an industrial system based on the cost free warranty policy. J. Braz. Soc. Mech. Sci. Eng. 2018, 40, 265. [CrossRef]

41. Garg, H. A novel approach for analyzing the reliability of series-parallel system using credibility theory and different types of intuitionistic fuzzy numbers. J. Braz. Soc. Mech. Sci. Eng. 2016, 38, 1021-1035. [CrossRef]

42. Garg, H. An efficient biogeography based optimization algorithm for solving reliability optimization problems. Swarm Evol. Comput. 2015, 24, 1-10. [CrossRef]

43. Garg, H.; Rani, M.; Sharma, S.P. Preventive maintenance scheduling of pulping unit in a paper mill. Jpn. J. Ind. Appl. Math. 2013, 30, 397-414. [CrossRef]

(C) 2019 by the authors. Licensee MDPI, Basel, Switzerland. This article is an open access article distributed under the terms and conditions of the Creative Commons Attribution (CC BY) license (http:/ / creativecommons.org/licenses/by/4.0/). 